Check for updates

Cite this: Phys. Chem. Chem. Phys., 2017, 19, 24264

Received 20th June 2017,

Accepted 11th August 2017

DOI: $10.1039 / c 7 c p 04145 d$

rsc.li/pccp

\title{
Calix[n]arene-based polyradicals: enhancing ferromagnetism by avoiding edge effects $\dagger$
}

\author{
Daniel Reta, (D)*ab Ibério de P. R. Moreira (iD ${ }^{a}$ and Francesc Illas (D) ${ }^{a}$
}

\begin{abstract}
Through-bond interacting organic polyradicals, rendered by customizable capacities of the state-of-theart synthetic routes, are ideal systems to investigate spin topologies. Relying on Rajca and co-workers' synthetic efforts, hereby we investigate the role of borders in the stability of the high-spin ground state in a series of realistic linear and ring-like arylmethyl polyradical derivatives. We show that, compared to their linear counterpart, the absence of borders in a ring-like arrangement of arylmethyl radicals imposes a larger number of spin-alternation rule violations, which strongly stabilizes the high-spin ground state. In addition, the structural flexibility of the investigated compounds translates into the existence of various structural energy minima for which the ferromagnetic ground state is always maintained. In view of the present results we propose these rings as possible candidates for the development of enhanced high spin single molecule toroics.
\end{abstract}

\section{Introduction}

Ever since the synthesis of the triphenylmethyl radical by Gomberg, ${ }^{1}$ carbon-based $\pi$-conjugated polyradicals interacting through-bond have been considered as candidates for the practical realization of purely organic magnetism. Among the different coupling schemes enabling one to obtain extended structures from triarylmethyl-based building blocks, only a 1,3 (or meta)-connectivity between the radical centres ensures a preferential high-spin ground state, in agreement with wellknown rules derived from topological arguments. ${ }^{2-5}$ This structural constraint in assembling the building blocks results in a limited number of possible structures such as linear, dendritic, star-branched or macrocyclic architectures, which indeed are expected to determine the macroscopic properties of the compounds. ${ }^{6-8}$

Fig. 1 schematically summarizes the most significant experimental achievements obtained by exploiting a 1,3 (or meta)connectivity using triarylmethyl-based radicals and classifies them as a function of the measured multiplicity of the ground state $v s$. relative stability. Here, relative stability is not a welldefined experimental property, but rather used for qualitatively comparing the most stable of these radicals. Fig. 1 also indicates the relationship between the adopted coupling scheme and the

\footnotetext{
${ }^{a}$ Departament de Ciència de Materials i Química Física \& Institut de Química Teòrica i Computacional (IQTCUB), Universitat de Barcelona, C/Martí i Franquès 1, 08028 Barcelona, Spain

${ }^{b}$ School of Chemistry, The University of Manchester, Oxford Road, Manchester, M13 9PL, UK. E-mail: daniel.reta@manchester.ac.uk

$\dagger$ Electronic supplementary information (ESI) available. See DOI: 10.1039/ c7cp04145d
}

associated deficiencies. One of the main issues here is the lack of chemical stability of the carbon-based radicals, with dimerization, or other types of reactions, likely to result in loss of any magnetic property of interest. To protect the polyradical character of these compounds, two main synthetic strategies emerged. One is based on the idea that larger conjugation would result in a more pronounced delocalization of the unpaired electrons making them less reactive. Rajca and co-workers have led this branch with the refinement of the carbanion method and the development of spin clusters; ${ }^{9-11}$ see the lower part of Fig. 1. The second approach aims at sterically protecting the radical centre by introducing bulky chlorine atoms, resulting in the well-known perchlorotriphenylmethyl (PTM) radical originally synthesized by Ballester et al. ${ }^{12}$ Based on this, Veciana and coworkers have managed to synthesize $\mathrm{di}^{-13}$ and triradicals ${ }^{14}$ showing respectively stable triplet and quartet states in solution at room temperatures, as well as to synthesize and characterize a large series of multifunctional materials; ${ }^{15-19} c f$. the upper part of Fig. 1 . Unfortunately, one cannot simultaneously take advantage of the two strategies simply because they are mutually exclusive due to steric congestion. $^{20}$ As a result, no major experimental advance has been pursued in the field in the last decade. It is at this point when one can envisage exploiting the inherent structural flexibility $^{21}$ present in these compounds so as to propose alternative approaches combining both a large conjugation and steric protection of the radical centres.

Relying on the ideas outlined above, it has been recently shown that a linear triarylmethyl-based polyradical molecule adopts a more stable helical conformation, which also promotes larger ferromagnetic interactions. ${ }^{22}$ However, the presence of 


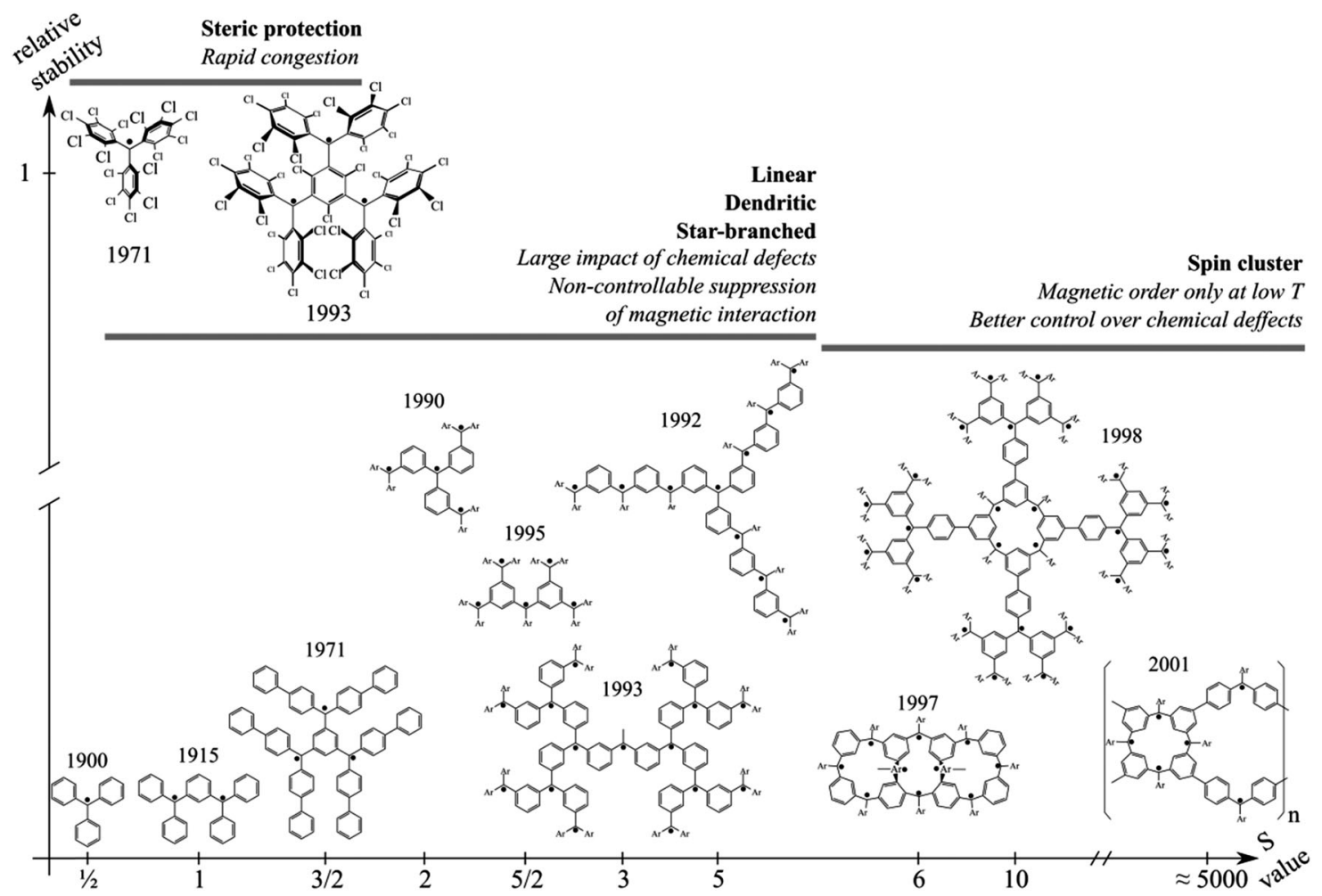

Fig. 1 Schematic representation of representative experimental achievements in the field of triarylmethyl-based radicals. Different experimental approaches for the assembly of the building blocks and associated deficiencies found in the synthesis are indicated with bold and italic letters, respectively. Note that for the "1993" molecule, chemical defects result in smaller multiplicity values than expected, which triggered the development of spin-clusters. Black dots in the molecules represent unpaired electrons. Ar- stands for the aromatic ring; $\mathrm{Cl}$ - for the chlorine atom.

edges in these high-spin ground state linear-like systems plays a detrimental role in ferromagnetism. This can be understood by simple arguments derived from the spin alternation rule schematically depicted in Fig. 2. Starting from a high spin arrangement with all spins up, the occurrence of a spin-flip in any of the radical centres leads to a situation that disrupts the spin-alternation rule forcing a spin frustration and a concomitant penalizing suppression of spin density in the adjacent phenyl rings. From theoretical and computational points of view, such a situation with one (or more) spin-flip can be represented by an antiferromagnetic solution (AFM). It is clear that the only region of the polyradical chain, either in a linear or helical conformation, where a spin flip imposes a minimum amount of disruptions is precisely at the edge. Consequently, the corresponding AFM solution will be stabilized which, again, is detrimental to an energetically isolated high spin ground state. Thus, to maintain a stable, ferromagnetic, high spin ground state, one should be able to equally penalize all AFM solutions and this is possible if one can get rid of the borders.

It is obvious that the most straightforward manner to remove edge effects in finite systems is by generating a ring-like structure. Inorganic chemistry has provided extraordinary relevant examples, ${ }^{23-33}$ but the localized nature of the magnetic centres and the presence of necessary bridging ligands often result in low-spin ground states and weak to moderate antiferromagnetic interactions. In organic chemistry, on the other hand, remarkable examples of ring-like molecules ${ }^{34-44}$ in the field of host-guest and

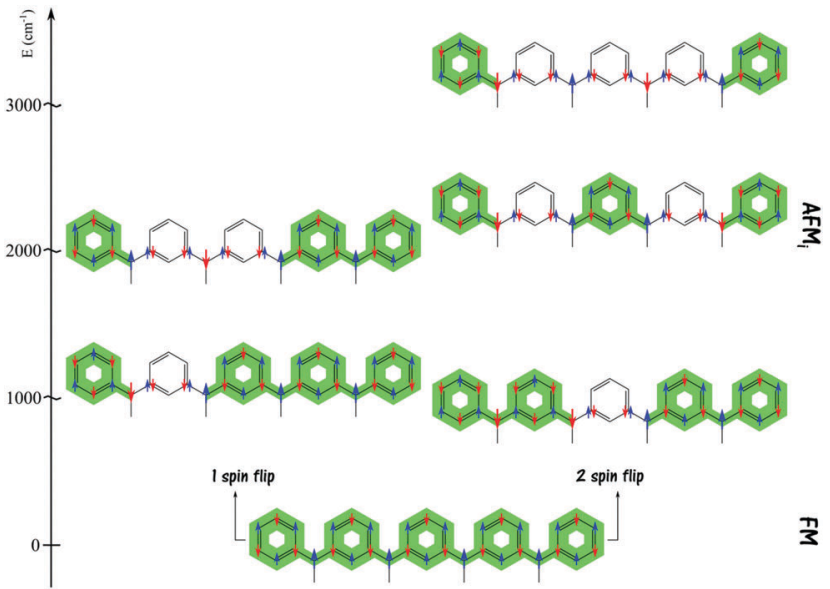

Fig. 2 Representation of different antiferromagnetic (AFM) solutions and associated relative energies that can be obtained from the high-spin ground state by applying one or two spin flips (left or right, respectively), in a $N=4$ system. Blue and red arrows stand for spin-up and down respectively. Coloured regions indicate the existence of delocalized spin density due to the fulfilment of the spin alternation rule. A spin-flip in one radical centre at the extreme (middle) disrupts the spin density in one (two) bridging ring, with a concomitant $\sim 10^{3} \mathrm{~cm}^{-1}\left(\sim 2 \times 10^{3} \mathrm{~cm}^{-1}\right)$ destabilization. Note, that in a ring, the lack of edges prevents the existence of low-spin solutions with only one disruption.

supramolecular chemistry have been achieved. However, attempts to obtain organic magnetic rings are scarce. For the purpose of the 
present work, the most relevant example is precisely the tetraradical that led Rajca and co-workers to coin the spin cluster term. $^{45}$ Despite their success, no further attempts have been reported to achieve more extended rings with a larger number of interacting radical centres, as the interest shifted towards the linkage of the tetraradical ring units. ${ }^{7}$ Other unrelated approaches consist of planar systems where the polyradical character arises from an equilibrium between quinoidal and aromatic forms, ${ }^{46,47}$ and of circular covalent organic frameworks (COFs) to which stable nitronyl radicals are covalently bound. ${ }^{48}$ Of particular significance for the present work is the concept of single molecule toroics (SMT) ${ }^{32}$ where on top of exchange interactions between centres, having a significant magnetic anisotropy is a key goal. Despite the fact that magnetic anisotropy is not expected in purely organic molecules, appropriate coordination with metallic centres appears as an effective manner to introduce such a property. ${ }^{49,50}$

Bearing in mind the structural flexibility of arylmethylderivatives and aiming at further investigating them as the most promising candidates to develop organic magnetism, we study a series of realistic, progressively bigger, circular-like, arylmethyl-based polyradicals. We then compare the magnetic features of these ring-like compounds to those of their associated linear and helical counterparts. Through a systematic theoretical study of their structural and magnetic features, we provide compelling evidence that a ring-like arrangement persistently presents comparatively more stable high-spin ground states, which is not affected by the ease to undergo conformational distortions. Additionally, the chemical stability of the investigated examples could be largely increased by promoting a favourable balance between steric protection and the associated strain.

\section{Computational and theoretical details}

All calculations have been carried out with the Gaussian09 suite of programs, ${ }^{51}$ using the $\mathrm{B}^{2} \mathrm{LYP}^{52}$ hybrid density functional within the unrestricted formalism and the Pople-type basis set 6-31G(d,p $)^{53-55}$ for all atoms, including dispersion corrections as proposed by Grimme. ${ }^{56-58}$ All discussed structures have been fully optimized and characterized as stationary minima in the potential energy surface (PES) by explicitly calculating the hessian matrix and making sure that all frequencies were positive and the forces (numerically) zero. For $N \geq 12$ systems ( $N$ is the number of radical centres), explicit calculation of hessian was not possible due to memory problems; however they fulfilled the convergence criteria in the optimization cycle. For complicated cases, the optimized geometry obtained using the semi-empirical method PM6 ${ }^{59}$ was used as a starting point for the B3LYP method. A further assessment of the employed method has been done by comparing the predicted structures and energy differences to different functionals and basis sets, for the 7-membered ring as a representative case. Thus, we selected the hybrid TPSS $^{60}$ meta-GGA functional, PBE0 ${ }^{61}$ and
M06- $2 \mathrm{X}^{62}$ hybrid functionals and the LC- $\omega \mathrm{PBE}^{63-65}$ long rangecorrected functional and the cc-pVTZ ${ }^{66}$ basis set.

To gain information about the magnitude of energy change induced by spin flips from the high spin state a common strategy consists in mapping the low energy ferromagnetic and antiferromagnetic solutions to the corresponding expectation values of a given spin only model Hamiltonian. Most often the well-known Heisenberg-Dirac-Van Vleck (HDVV) model Hamiltonian as in eqn (1) is chosen

$$
\hat{H}^{\mathrm{HDVV}}=-\sum_{\langle i, j\rangle} J_{i j} \hat{\boldsymbol{S}}_{i} \cdot \hat{\boldsymbol{S}}_{j}
$$

where $J_{i j}$ is the exchange coupling constant between the $\hat{\boldsymbol{S}}_{i}$ and $\hat{\boldsymbol{S}}_{j}$ localized spin moments and the $\langle i, j\rangle$ symbol indicates that the sum refers to the nearest neighbour interactions only. Nevertheless, for the purpose of this work, the important quantity is the total energy difference between the ferro- and lowest in energy antiferromagnetic solutions, which is independent of the adopted model spin Hamiltonian. For completeness, details of a possible mapping ${ }^{67-70}$ to the HDVV are given in the ESI. $\dagger$

\section{Linear, helical and circular oligomers: structure and magnetism}

In order to analyse the impact of edge effects on the relative stability of FM solutions, we compare a series of oligomers in a linear, helical and ring conformation ranging from 4 to 15 radical centres. The linear and helical structures contain exactly the same number and type of atoms and therefore one can make use of absolute energies to discuss the relative properties. However, the ring arrangement possesses one less phenyl moiety and the discussion is then referred to relative energy differences per magnetic centre. For completeness, we also investigated the steric protection of the radical centres in some of the rings, by substituting the hydrogen atoms in the radicalbearing carbon by phenyl rings and in a subsequent step (for the smallest ring), by substituting all hydrogen atoms by chlorine atoms.

Let us first discuss the results obtained for the linear and helical structures. Table 1(a) presents the energy difference between the FM ground state and the lowest (excited) AFM solution for the linear and helical conformations. As predicted by the simple topological arguments discussed in Fig. 2, it is found that the lowest AFM solutions always correspond to the arrangement of consecutive spin down densities at one of the edges (compare $\mathrm{AFM}_{(i)}$ and $\mathrm{AFM}_{(i)}$ rows in Tables S2 and S3 in the ESI $\dagger)$. This follows from the fact that this type of AFM solution involves the minimum amount of spin alternation rule violations. Due to technical limitations on the control of the topology of the solution sought for, the solution presenting consecutive spin down densities at the edge was not possible to converge in a few cases (helical $N=8,10,13$ and 14) despite extensive efforts made using different starting density and convergence control procedures. In any case, all converged 
Table 1 Energy differences (in $\mathrm{cm}^{-1}$ ) between the FM ground state and lowest excited AFM solutions, calculated per number of magnetic centres $(N)$ in each of the linear, helical and ring minima. (a) $\Delta E_{\mathrm{L}-\mathrm{H}}$ column shows

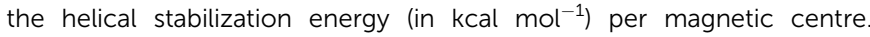
* indicates that solutions with all consecutive spin-down in one of the chain extremes were not converged. (b) The different characterized minima are indicated in bold. $\Delta E_{\mathrm{FM}^{i}-\mathrm{FM}}{ }^{j}$ column shows the energy difference (in $\mathrm{kcal} \mathrm{mol}^{-1}$ ) between two $i$ and $j$ minima for a given ring with $N$ magnetic centres, being minimum 1 the lowest in energy

\begin{tabular}{|c|c|c|c|c|c|c|c|c|c|}
\hline \multirow[b]{2}{*}{$N$} & \multicolumn{2}{|c|}{ (a) $\Delta E_{\mathrm{FM}-\mathrm{AFM}}$} & \multirow{2}{*}{$\Delta E_{\mathrm{L}-\mathrm{H}}$} & \multicolumn{4}{|c|}{ (b) $\Delta E_{\mathrm{FM}-\mathrm{AFM}}$} & \multicolumn{2}{|c|}{$\Delta E_{\mathrm{FM}^{i}-\mathrm{FM}} j$} \\
\hline & Linear & Helical & & 1 & 2 & 3 & 4 & $1 \rightarrow 2$ & $1 \rightarrow 31 \rightarrow 4$ \\
\hline 4 & -291 & -175 & 2.3 & -572 & & & & & \\
\hline 5 & -283 & -165 & 3.1 & -387 & -454 & -353 & & -8.2 & -17.0 \\
\hline 6 & -194 & -86 & 3.6 & -367 & -27 & & & -17.5 & \\
\hline 7 & -167 & -88 & 4.1 & -266 & -203 & & & -35.2 & \\
\hline 8 & -145 & * & 4.4 & -283 & -270 & -269 & -209 & -1.3 & $-24.0-34.2$ \\
\hline 9 & -130 & -51 & 4.7 & -228 & -205 & -184 & -213 & -1.0 & $-6.6 \quad-17.4$ \\
\hline 10 & -117 & * & 4.9 & -205 & -233 & -180 & & -1.1 & -25.6 \\
\hline 12 & -97 & -44 & 5.2 & -178 & & & & & \\
\hline 13 & -90 & * & 5.3 & & & & & & \\
\hline 14 & -83 & * & 5.4 & & & & & & \\
\hline 15 & & & & -133 & & & & & \\
\hline
\end{tabular}

solutions are presented in Table S3 (ESI $\dagger$ ) thus providing a strong support to the conclusions reached in the present work. Table 1(a) also indicates the gain in stability (kcal mol ${ }^{-1}$ ) per magnetic centre due to the appearance of the helical conformation. As indicated before, the expressions to obtain the magnetic couplings can be found in Section 1 of the ESI. $\dagger$ For the linear molecules (Table S4 and Section 3 of the ESI $\dagger$ ), the calculated exchange coupling constants remain practically the same throughout the series, where $J_{1}$ is an order of magnitude larger than $J_{2}$ and of opposed sign. The situation is less clear for the helical arrangement (Table S4 and Section 3 of the ESI $\dagger$ ), as there is a significant variation in the magnitude and sign of the different two body terms with the number of centres. Additionally, depending on which DFT-computed energies are used to solve the spectrum, one finds a large variation in $J_{3}$ and $J_{4}$ (see Section 3 in the ESI $\dagger$ ). The crucial point here is, however, the consistent prediction of a helical-induced stabilization of the FM ground state in all cases that can be safely addressed considering the HDVV spin model Hamiltonian to classify and compare the different spin solutions.

Using the data in Table 1(b), we focus now on the results for the ring-like structures. Table 1 (b) presents (i) the energy differences between the ground ferromagnetic and the lowest AFM solutions (which correspond to consecutive beta-centres) at each of the different local minima found and (ii) the energy cost to change conformation among the different minima. Concerning the lowest energy structures, Fig. 3a depicts the corresponding geometries of four representative cases displaying $N=4,7,10$ and 15 magnetic centres (see Table S5 in the ESI $\dagger$ for absolute energies associated with FM and AFM). The cases corresponding to $N=4$ and $N=10$ are of particular relevance due to reported experimental information. Thus, for $N=4$ the optimized structure shows a 4-fold-symmetry, as experimentally found for phenyl-substituted calix[4]arene, ${ }^{45}$ and for $N=4$ and 10 the adopted conformation of the inner rings resemble the ones reported for related closed-shell aza $\left.1_{n}\right]$ metacyclophanes. ${ }^{44}$ The $N=4$ system also allows exemplifying the spin density associated with the FM and AFM solutions, as indicated in Fig. 3b. From this simple case, the spin densities of all possible AFM solutions for larger systems can be envisioned. As implicit in the previous discussion, these systems present an additional complexity
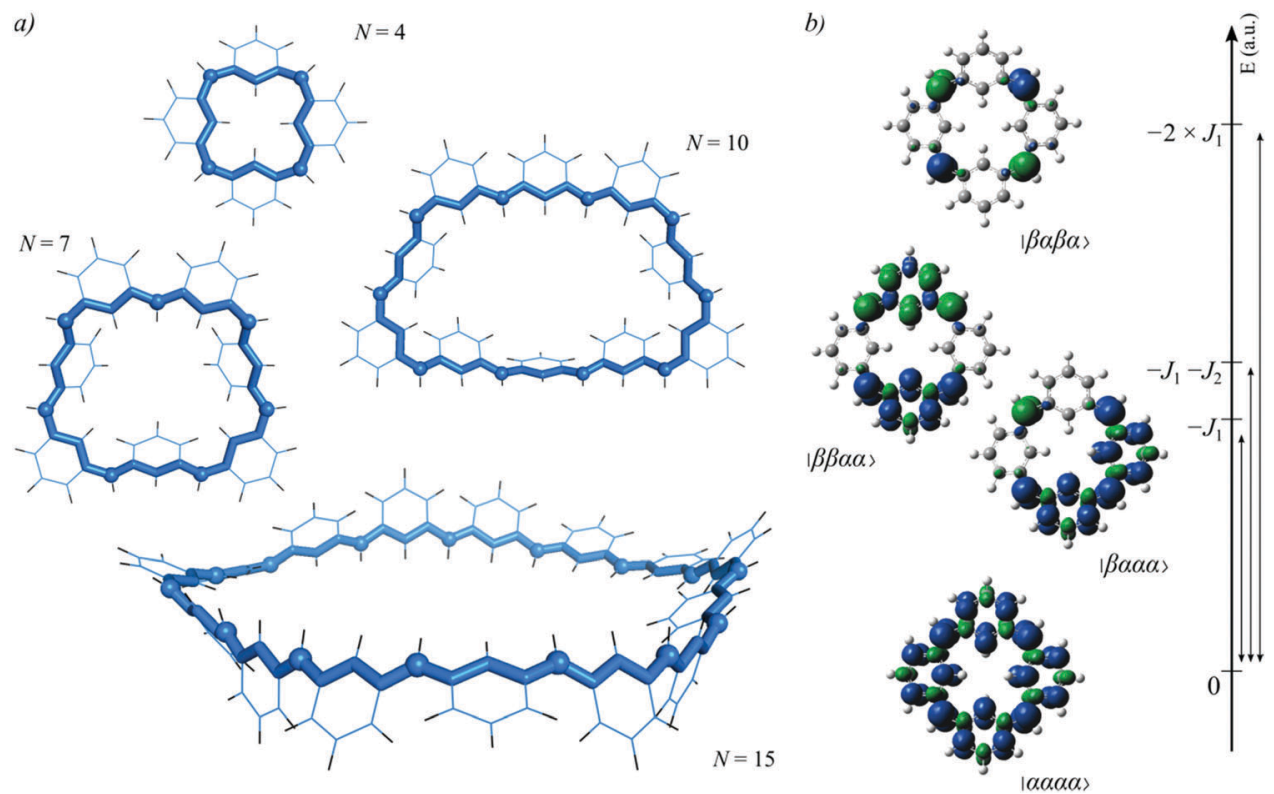

Fig. 3 (a) Representation of the molecular geometries associated with the lowest energy minima found for the $N=4,7,10,15$ representative cases ( $N$ number of $S=1 / 2$ centres). Carbon-based radical centres are highlighted using a ball representation. Carbon and hydrogen atoms are depicted in blue and black, respectively. (b) Spin density plots and associated mapping of FM, AFM $1, A F M_{2}$ and $A F M_{2 *}$ solutions for $N=4$ ring. Blue and green colours indicate $\alpha$ and $\beta$ spin densities. Similar spin densities are to be associated with the different solutions presented for the larger rings $\left(J_{1} \sim 2000 \mathrm{~cm}^{-1}\right)$. 
arising from the multiple local minima that a ring structure can adopt. For instance, for $N=8$ a total of four, well separated in energy, minima were characterized. Interestingly, for the largest molecular structure investigated $(N=15)$, the energy minimum structure is reminiscent of a Möbius strip and, despite the different degree of strain found in the different minima, the ground state always remains FM. This strongly supports the claim that these systems exhibit a robust FM ground state, a conclusion which, qualitatively speaking, can be considered independent of the DFT based-method used. In fact, the validity of the B3LYP functional with the standard Pople-type GTO basis set to describe these types of organic radicals has been extensively validated in previous theoretical ${ }^{21,71}$ and experimental ${ }^{72,73}$ works. Taken altogether, one can safely conclude that a more exhaustive investigation of the potential energy surfaces will not reveal a qualitatively different picture in terms of magnetic interactions and that the presented cases are sufficiently representative. To further justify this, Table S11 in Section 5 of the ESI $\dagger$ presents the results obtained with TPSS, PBE0, M06-2X and LC- $\omega$ PBE functionals together with the $6-31 G(d, p)$ and triple- $\zeta$ polarized quality basis sets, for the 7-membered ring system. As shown for the $m$-xylylene diradical, ${ }^{71}$ the long-range separated functional predicts values off the trends, while the rest of the functionals behave in a more consistent way.

Having separately discussed linear-like and ring-like structures we now compare the results obtained for the two types of structures. For a given number of radical centres, the energy difference between the FM and lowest AFM solutions is always larger for the ring, as compared to either linear and helical arrangements; this proves that avoiding edge effects provides a clear strategy to stabilize high spin states in triarylmethyl polyradical derivatives. In fact, a single spin flip anywhere in the ring results in a state approximately $2400 \mathrm{~cm}^{-1}$ above the FM ground state, which is twice the difference found in the linear cases (see $\mathrm{AFM}_{1}$ rows in Table S2 vs. Table S5 in the ESI $\dagger$ ). Interestingly, due to spin topology, while removing edge effects significantly stabilizes the FM solution, the magnetic coupling constant value $J_{1}$ remains similar to the linear counterpart. This can be understood by comparing eqn (S2) and (S6) in the ESI. $\dagger$ However, $J_{2}$ shows a considerable variation along the series. It is worth mentioning that, as in the case of the helical structures, the calculated magnetic coupling constants also show differences depending on which set of equations is used (Table S7 in Section 4 of the ESI $\dagger$ ) which seem to indicate that the HDVV Hamiltonian used is a too crude spin model for this type of system. In this case, the apparent inconsistency can be traced to the rather irregular distribution of radical centres within the molecule, which makes the distances between the first and second nearest neighbours not always constant. The impact of the adopted conformation on the magnetic coupling constants is further investigated by calculating those values at the different conformers, as presented in Table S8 of the ESI. $\dagger$ For all investigated cases, $J_{1}$ remains largely ferromagnetic although in some cases it experiences a noticeable decrease. On the other hand, $J_{2}$ does switch from ferro- to antiferrocharacter depending on the conformation. Nevertheless, $J_{1}$ is, in all cases, one order of magnitude larger than $J_{2}$, thus retaining the dominant magnetic interaction. To conclude the study, a similar analysis was carried out on more realistic molecules where the steric protection of the radical centres is increased by (i) substituting the hydrogen atom in each carbon-based radical by a phenyl ring for $N=4,5,7,8$ or (ii) substituting all hydrogen atoms by chlorine atoms for $N=4$ (see Table S9 in the ESI $\dagger$ ). The general trends are the same but the absolute values of the magnetic coupling constants are smaller, due mainly to a larger delocalization of the unpaired spin density on the rings that do not participate in the exchange coupling. For instance, $J_{1}$ drops from 2333 to $1637 \mathrm{~cm}^{-1}$ for the lowest stationary point found for $N=8$ after replacing the hydrogen atoms in the radical centre by phenyl groups. Additionally, the fully chlorinated $N=4$ case also shows a ferromagnetic ground state in both stationary points found, despite presenting a smaller $J_{1}$ value. Finally, the relative energy position of the molecular orbitals does not depend on the size of the molecule and the steric protection of the radical centres does not significantly modify the HOMO-LUMO gap, which remains around $2 \mathrm{eV}$ (see Fig. $\mathrm{S} 2$ in the $\mathrm{ESI}^{\dagger}$ ). This is in contrast with what is predicted for planar arrangements of triarylmethylbased polyradicals. ${ }^{74}$ Nevertheless, the important finding is that, irrespective of the HDVV used to map the different magnetic solution, removing edges significantly stabilizes the FM ground state, strongly suggesting that this may be a potential way to obtain polyradicals with robust ferromagnetism.

\section{Conclusions}

The present study shows that structurally stable, ring-like molecules derived from arylmethyl polyradicals display largely stabilized high-spin ground states as compared to their linearlike counterparts. The reason is the absence of borders, as predicted by simple spin topological arguments. Rajca and co-workers $^{45}$ exploited the basic calix[4] arene to develop spin clusters through the linkage of these units, but syntheses of larger rings have not been reported.

A meta-connectivity of the radical centres is crucial for two reasons: (i) it ensures high-spin ground states and (ii) offers a structural freedom, increasing with size, that alleviates possible strains imposed by the ring structures. In fact, the inner ring of all optimized structures presented in this work resembles previous experimentally reported geometries in related closedshell aza[ $\left.1_{n}\right]$ metacyclophanes, ${ }^{44}$ which indicates the feasibility of the proposed molecules.

For ring structures with a given number of centres, several local structural minima are found. The preference for a highspin ground state, the increased energy difference between FM and AFM solutions and the predicted HOMO-LUMO gap are not altered by the conformational richness. The calculated nearest neighbour $\left(J_{1}\right)$ coupling remains always the dominant term and exhibit robust ferromagnetic character.

Further stabilization of the reactive carbon-based radical centres can be achieved by steric protection, maintaining the 
main structural and magnetic characteristics. The ideal situation would then be to find a balance between steric congestion and stabilization of the radical centres.

To summarize, removal of edges by considering purely organic, through-bond interacting, meta-connected ring-like molecules derived from arylmethyl polyradicals appears as a realistic strategy to obtain very high-spin systems and move forward in the field of organic magnetism. Particularly, the investigated rings can be considered as potential candidates for the development of enhanced single-molecule toroics (SMT), ${ }^{32}$ owing to their extended conjugation within the molecule and the large ferromagnetic exchange interactions. Taken together, these findings call for efforts to attempt the synthesis of ring-like arylmethylbased molecules, along a well-defined synthetic route.

\section{Conflicts of interest}

There are no conflicts to declare.

\section{Acknowledgements}

This research was supported by the Spanish MINECO/FEDER CTQ2015-64618-R and CTQ2016-76423-P grants and, in part, by Generalitat de Catalunya (grants 2014SGR97 and XRQTC) and by the NOMAD Center of Excellence project, which received funding from the European Union's Horizon 2020 research and innovation programme under grant agreement No 676580 . DR acknowledges additional funding from the Engineering and Physical Sciences Research Council (EP/P002560/1 grant). FI acknowledges additional support from the 2015 ICREA Academia Award for Excellence in University Research. The toroid of the image in table of content was created by Yassine Mrabet. This vector image was created with Inkscape. Under GFDL and CC BY-SA 3.0, via Wikimedia Commons.

\section{References}

1 M. Gomberg, J. Am. Chem. Soc., 1900, 22, 757-771.

2 H. C. Longuet-Higgins, J. Chem. Phys., 1950, 18, 265-274.

3 A. A. Ovchinnikov, Theor. Chim. Acta, 1978, 47, 297-304.

4 W. T. Borden and E. R. Davidson, J. Am. Chem. Soc., 1977, 99, 4587-4594.

5 N. Mataga, Theor. Chim. Acta, 1968, 10, 372-376.

6 A. Rajca, Chem. Rev., 1994, 94, 871-893.

7 A. Rajca, Adv. Phys. Org. Chem., 2005, 40, 153-199.

8 N. M. Shishlov, Russ. Chem. Rev., 2007, 75, 863-884.

9 A. Rajca, K. Lu and S. Rajca, J. Am. Chem. Soc., 1997, 119, 10335-10345.

10 S. Rajca, A. Rajca, J. Wongsriratanakul, P. Butler and S. M. Choi, J. Am. Chem. Soc., 2004, 126, 6972-6986.

11 A. Rajca, J. Wongsriratanakul and S. Rajca, Science, 2001, 294, 1503-1505.

12 M. Ballester, J. Riera-Figueras, J. Castaner, C. Badfa and J. M. Monso, J. Am. Chem. Soc., 1971, 93, 2215-2225.
13 J. Veciana, C. Rovira, M. I. Crespo, O. Armet, V. M. Domingo and F. Palacio, J. Am. Chem. Soc., 1991, 113, 2552-2561.

14 J. Veciana, C. Rovira, N. Ventosa, M. I. Crespo and F. Palacio, J. Am. Chem. Soc., 1993, 115, 57-64.

15 D. Maspoch, D. Ruiz-Molina and J. Veciana, Chem. Soc. Rev., 2007, 36, 770-818.

16 N. Crivillers, M. Mas-Torrent, J. Vidal-Gancedo, J. Veciana and C. Rovira, J. Am. Chem. Soc., 2008, 130, 5499-5506.

17 M. Mas-Torrent, N. Crivillers, C. Rovira and J. Veciana, Chem. Rev., 2012, 112, 2506-2527.

18 C. Simão, M. Mas-Torrent, N. Crivillers, V. Lloveras, J. M. Artés, P. Gorostiza, J. Veciana and C. Rovira, Nat. Chem., 2011, 3, 359-364.

19 I. Ratera and J. Veciana, Chem. Soc. Rev., 2012, 41, 303-349.

20 D. Ruiz-Molina, J. Veciana, F. Palacio and C. Rovira, J. Org. Chem., 1997, 62, 9009-9017.

21 D. Reta Mañeru, I. de P. R. Moreira and F. Illas, Theor. Chem. Acc., 2015, 134, 18.

22 D. Reta Mañeru, I. de P. R. Moreira and F. Illas, J. Am. Chem. Soc., 2016, 138, 5271-5275.

23 M. L. Baker, G. A. Timco, S. Piligkos, J. S. Mathieson, H. Mutka, F. Tuna, P. Kozlowski, M. Antkowiak, T. Guidi, T. Gupta, H. Rath, R. J. Woolfson, G. Kamieniarz, R. G. Pritchard, H. Weihe, L. Cronin, G. Rajaraman, D. Collison, E. J. L. McInnes and R. E. P. Winpenny, Proc. Natl. Acad. Sci. U. S. A., 2012, 109, 19113-19118.

24 M. Affronte, T. Guidi, R. Caciuffo, S. Carretta, G. Amoretti, J. Hinderer, I. Sheikin, A. G. M. Jansen, A. A. Smith, R. E. P. Winpenny, J. van Slageren and D. Gatteschi, Phys. Rev. B: Condens. Matter Mater. Phys., 2003, 68, 104403.

25 O. Cador, D. Gatteschi, R. Sessoli, A.-L. Barra, G. A. Timco and R. E. P. Winpenny, J. Magn. Magn. Mater., 2005, 290, 55-60.

26 G. N. Newton, N. Hoshino, T. Matsumoto, T. Shiga, M. Nakano, H. Nojiri, W. Wernsdorfer, Y. Furukawa and H. Oshio, Chem. - Eur. J., 2016, 22, 14205-14212.

27 R. E. P. Winpenny, G. A. Timco, S. Marocchi, E. Garlatti, M. Albring, V. Bellini, F. Manghi, E. J. L. McInnes, R. Pritchard, F. Tuna, W. Wernsdorfer, G. Lorusso, G. Amoretti, S. Carretta and M. Affronte, Dalton Trans., 2016, 45, 16610-16615.

28 T. Minato, K. Suzuki, K. Yamaguchi and N. Mizuno, Angew. Chem., Int. Ed., 2016, 55, 9630-9633.

29 S. Sanz, J. M. Frost, T. Rajeshkumar, S. J. Dalgarno, G. Rajaraman, W. Wernsdorfer, J. Schnack, P. J. Lusby and E. K. Brechin, Chem. - Eur. J., 2014, 20, 3010-3013.

30 J. van Slageren, S. Piligkos and F. Neese, Dalton Trans., 2010, 39, 4999-5004.

31 P. Santini, S. Carretta, G. Amoretti, T. Guidi, R. Caciuffo, A. Caneschi, D. Rovai, Y. Qiu and J. R. D. Copley, Phys. Rev. B: Condens. Matter Mater. Phys., 2005, 71, 184405.

32 L. Ungur, S.-Y. Lin, J. Tang and L. F. Chibotaru, Chem. Soc. Rev., 2014, 43, 6894-6905.

33 V. Chandrasekhar, S. Biswas, S. Das, J. Acharya, V. Kumar, J. Leusen, J. M. Herrera, P. Koegerler and E. Colacio, Chem. - Eur. J., 2017, 23, 5145-5170.

34 A. Ikeda and S. Shinkai, Chem. Rev., 1997, 97, 1713-1734. 
35 N. Morohashi, F. Narumi, N. Iki, T. Hattori and S. Miyano, Chem. Rev., 2006, 106, 5291-5316.

36 D. R. Stewart and C. D. Gutsche, J. Am. Chem. Soc., 1999, 121, 4136-4146.

37 C. D. Gutsche, Calixarenes, Royal Society of Chemistry, Cambridge, 2008.

38 F. Chen, T. Tanaka, Y. Hong, W. Kim, D. Kim and A. Osuka, Chem. - Eur. J., 2016, 22, 10597-10606.

39 Z.-A. Huang, C. Chen, X.-D. Yang, X.-B. Fan, W. Zhou, C.-H. Tung, L.-Z. Wu and H. Cong, J. Am. Chem. Soc., 2016, 138, 11144-11147.

40 T. Josse, J. De Winter, P. Gerbaux and O. Coulembier, Angew. Chem., Int. Ed., 2016, 55, 13944-13958.

41 T. Kim, N. Singh, J. Oh, E.-H. Kim, J. Jung, H. Kim and K.-W. Chi, J. Am. Chem. Soc., 2016, 138, 8368-8371.

42 G. Trinquier and J.-P. Malrieu, Chem. - Eur. J., 2015, 21, 814-828.

43 T. Ogoshi, T. Yamagishi and Y. Nakamoto, Chem. Rev., 2016, 116, 7937-8002.

44 M. Vale, M. Pink, S. Rajca and A. Rajca, J. Org. Chem., 2008, 73, 27-35.

45 A. Rajca, S. Rajca and S. R. Desai, J. Am. Chem. Soc., 1995, 117, 806-816.

46 S. Nobusue, H. Miyoshi, A. Shimizu, I. Hisaki, K. Fukuda, M. Nakano and Y. Tobe, Angew. Chem., Int. Ed., 2015, 54, 2090-2094.

47 S. Das, T. S. Herng, J. L. Zafra, P. M. Burrezo, M. Kitano, M. Ishida, T. Y. Gopalakrishna, P. Hu, A. Osuka, J. Casado, J. Ding, D. Casanova and J. Wu, J. Am. Chem. Soc., 2016, 138, 7782-7790.

48 B. K. Hughes, W. A. Braunecker, D. C. Bobela, S. U. Nanayakkara, O. G. Reid and J. C. Johnson, J. Phys. Chem. Lett., 2016, 7, 3660-3665.

49 T. Kusamoto, Y. Hattori, A. Tanushi and H. Nishihara, Inorg. Chem., 2015, 54, 4186-4188.

50 T. Kusamoto, S. Kimura and H. Nishihara, Chem. Lett., 2016, 45, 1057-1059.

51 M. J. Frisch, G. W. Trucks, H. B. Schlegel, G. E. Scuseria, M. A. Robb, J. R. Cheeseman, G. Scalmani, V. Barone, G. A. Petersson, H. Nakatsuji, X. Li, M. Caricato, A. Marenich, J. Bloino, B. G. Janesko, R. Gomperts, B. Mennucci, H. P. Hratchian, J. V. Ortiz, A. F. Izmaylov, J. L. Sonnenberg, D. Williams-Young, F. Ding, F. Lipparini, F. Egidi, J. Goings, B. Peng, A. Petrone, T. Henderson, D. Ranasinghe, V. G. Zakrzewski, J. Gao, N. Rega, G. Zheng, W. Liang, M. Hada, M. Ehara, K. Toyota, R. Fukuda, J. Hasegawa, M. Ishida, T. Nakajima, Y. Honda, O. Kitao, H. Nakai, T. Vreven, K. Throssell, J. A. Montgomery, Jr., J. E. Peralta, F. Ogliaro, M. Bearpark, J. J. Heyd, E. Brothers, K. N. Kudin,
V. N. Staroverov, T. Keith, R. Kobayashi, J. Normand, K. Raghavachari, A. Rendell, J. C. Burant, S. S. Iyengar, J. Tomasi, M. Cossi, J. M. Millam, M. Klene, C. Adamo, R. Cammi, J. W. Ochterski, R. L. Martin, K. Morokuma, O. Farkas, J. B. Foresman and D. J. Fox, Gaussian 09, Revision D.01, Gaussian, Inc., Wallingford CT, 2016.

52 A. D. Becke, J. Chem. Phys., 1993, 98, 5648-5652.

53 W. J. Hehre, R. Ditchfield and J. A. Pople, J. Chem. Phys., 1972, 56, 2257-2261.

54 J. D. Dill and J. A. Pople, J. Chem. Phys., 1975, 62, 2921-2922.

55 M. M. Francl, W. J. Pietro, W. J. Hehre, J. Stephen Binkley, M. S. Gordon, D. J. DeFrees and J. A. Pople, J. Chem. Phys., 1982, 77, 3654-3665.

56 S. Grimme, Wiley Interdiscip. Rev.: Comput. Mol. Sci., 2011, 1, 211-228.

57 S. Grimme, J. Comput. Chem., 2004, 25, 1463-1473.

58 S. Grimme, J. Comput. Chem., 2006, 27, 1787-1799.

59 J. J. P. Stewart, J. Mol. Model., 2007, 13, 1173-1213.

60 J. Tao, J. P. Perdew, V. N. Staroverov and G. E. Scuseria, Phys. Rev. Lett., 2003, 91, 146401.

61 C. Adamo and V. Barone, J. Chem. Phys., 1999, 110, 6158-6170.

62 Y. Zhao and D. G. Truhlar, Theor. Chem. Acc., 2008, 120, 215-241.

63 O. A. Vydrov and G. E. Scuseria, J. Chem. Phys., 2006, 125, 234109.

64 O. A. Vydrov, J. Heyd, A. V Krukau and G. E. Scuseria, J. Chem. Phys., 2006, 125, 74106-74109.

65 O. A. Vydrov, G. E. Scuseria and J. P. Perdew, J. Chem. Phys., 2007, 126, 154109.

66 T. H. Dunning, J. Chem. Phys., 1989, 90, 1007-1024.

67 I. de P. R. Moreira and F. Illas, Phys. Chem. Chem. Phys., 2006, 8, 1645-1659.

68 P. Rivero, I. de P. R. Moreira and F. Illas, J. Phys.: Conf. Ser., 2008, 117, 12025.

69 R. Maurice, R. Bastardis, C. de Graaf, N. Suaud, T. Mallah and N. Guihéry, J. Chem. Theory Comput., 2009, 5, 2977-2984.

70 I. de P. R. Moreira, C. J. Calzado, J. P. Malrieu and F. Illas, New J. Phys., 2007, 9, 369.

71 D. Reta Mañeru, A. K. Pal, I. D. P. R. Moreira, S. N. Datta and F. Illas, J. Chem. Theory Comput., 2014, 10, 335-345.

72 R. Gaudenzi, E. Burzurí, D. Reta, I. de P. R. Moreira, S. T. Bromley, C. Rovira, J. Veciana and H. S. J. van der Zant, Nano Lett., 2016, 16, 2066-2071.

73 R. Gaudenzi, J. de Bruijckere, D. Reta, I. de P. R. Moreira, C. Rovira, J. Veciana, H. S. J. van der Zant and E. Burzurí, ACS Nano, 2017, 11, 5879-5883.

74 I. Alcón, D. Reta, I. de P. R. Moreira and S. T. Bromley, Chem. Sci., 2017, 8, 1027-1039. 